\title{
Active and Supportive Computer-Mediated Resources for Student-to-Student Conversations
}

\author{
Noel Enyedy, Phil Vahey, and Bernard R. Gifford \\ Education of Mathematics, Science and Technology \\ Graduate School of Education, University of California at Berkeley
}

\begin{abstract}
Communication is a central aspect of human learning. Using the Probability Inquiry Environment (PIE) as an example, we examine how external representations (both textual and iconic) mediate face-to-face conversations among students, and support productive mathematical discourse. We provide quantitative data that suggests that seventh grade students who used PIE learned some of the basic principles of probability. Two cases studies are that illustrate how communication supported by computer-mediated representations contributed to this success. The first case study demonstrates how the computer can actively prompt student conversations that lead to learning. The second case study examines how an animated graphical representation supported these productive conversations.
\end{abstract}

\section{Introduction}

Computer Supported Collaborative Learning (CSCL) as a field of inquiry sets out to understand, support and change the learning practices of students and teachers working together in groups. This paper explores the theme of collaboration as a design principle and illustrates how to use the rich media of the computer to support students as they collaborate. Using the Probability Inquiry Environment (PIE) as an example, we look at the computer-mediated representational resources designed to help support productive mathematical conversations.

There is a synergetic relationship between the practices and artifacts of a community and an individual's thinking and activity. Clifford Geertz stated, "culture, rather than being added on, so to speak, to a finished or virtually finished animal, was ingredient, and centrally ingredient, in the production of the animal itself" (1973, p.47). Cognitive artifacts, those cultural artifacts used to aid the process of thinking and communication, quickly become inseparable from the knowledge and established practices of a community. While this relationship makes describing human activity increasingly difficult to analyze, it provides a leverage point to promote change. One way to conceptualize instructional design, then, is as a type of anthropological activism (diSessa personal communication) in which we leverage the relationship between tools and practice in an attempt to redefine the practices of the teaching and learning community through the introduction of new technologies.

Re-conceptualizing the issues of instructional design as anthropological activism encourages designers of educational software to seriously consider that a critical dimension of the design space is the practices and activities that surround and influence how the software will be used in a classroom. The purpose of this paper is to illustrate a genre of educational software that recognizes discourse practices as a central component of human learning and uses technology to support this key classroom practice and improve learning.

In this paper we will try to answer the question, "How can the computer be effectively used to start and support mathematical conversations?" Specifically, in the domain of probability, how can computermediated textual and graphical representations help students to articulate their naive intuitions about probability, and scaffold them in the process of constructing arguments that reflect a more standard understanding of probabilistic reasoning?

By examining the design of PIE and its implementation in a seventh grade classroom, we hope to refine both our theoretical understanding of collaboration and learning, and how to better support the emerging collaborative practices of the classroom. In this paper we first discuss the aspects of CSCL that influenced our initial design and then illustrate, through two case studies, how we refined the representational resources to make them better support collaborative learning. In the first example, we show how making the computer an active agent that prompted and structured student conversations created 
the opportunity for students to productively build on their existing ideas. In the second example, we explore how the students used a dynamic, graphical representation as a supporting resource to uncover the structure of the outcome space in a game of chance.

\section{The Role of Communication in Instruction and Learning}

CSCL is based on the premise that technology can be effectively used to mediate, support and redefine the interaction of students and teachers engaged in the teaching and learning enterprise (Koschmann et al. 1994). This belief, in turn, rests upon the well supported assumption that learning is intimately tied to the process of communication. However, communication is far too broad an idea to effectively guide instructional design-different situations and goals require different forms of communication. In fact, it has been argued that many of the problems our current educational system is experiencing can be directly traced to the fact that much of today's educational practices are based on a transmissive model of communication where the teacher attempts to impart knowledge to students by lecturing to them in a lock-step fashion (Pea, 1994; Gifford, 1997).

We posit that collaborative learning environments should be based on a transformative model of communication, defined by Roy Pea as conceptual learning conversations that lead to the social construction of understanding and individual conceptual change (1994). We believe that this view entails that learning environments support practices that allow students to: participate in authentic situations; be active in their learning; and reflect on, modify and articulate their understanding.

Additionally, we found it profitable to adopt the theoretical stance of distributed cognition to guide how we designed technologies to mediate and support transformative communicative practices, because this perspective carefully considers the interactions between the mind and the world in its analysis of intelligent activity. Distributed cognition holds that intelligence does not reside solely in the head of an individual but is an interactive process that is mutually constituted by the student, the symbolic and physical environment, and other social actors (Pea, 1993). As Edwin Hutchins points out technologies do not merely help us to accomplish tasks, they fundamentally change the nature of the task from the perspective of the user.

Mediating technologies do not stand between the user and the task. Rather, they stand with the user as resources used in the regulation of behavior in such a way that the propagation of representational state that implements the computation can take place...These tools permit the people using them to do the tasks that need to be done while doing the kinds of things people are good at: recognizing patterns, modeling simple dynamics of the world, and manipulating objects in the environment. (Hutchins, 1995, p. 154-155)

\section{Resources for Communication and Collaboration}

PIE uses the technology to mediate the learning process in three primary ways. First, the technology is used to create authentic investigations - we have discovered that having the students analyze the fairness of games of chance is an activity that students consider authentic, and leads to students spontaneously employing aspects of probability theory (Vahey, 1995). By authentic we mean that PIE engages the students in truly problematic situations. This follows Jean Lave's proposed use of the term, "Given lively imaginations, it does not matter whether the problems conform to life experience, but it is important that they engage the imagination, that they become really problematic" (1992). Although important to the success of PIE, this paper will not concentrate on authenticity, and will instead discuss the other aspects more directly related to the form and content of student discourse.

Second, the technology takes an active role in shaping specific interactions and practices, by prompting students to discuss the salient aspects of the domain during the investigation. By stopping the flow of activity and initiating a conversation on a particular topic, the computer takes on a social role in the interaction, either beginning or changing the direction of the students' conversation.

Third, the software provides non-linguistic resources that can productively support mathematical discourse by providing public reference objects. By public reference objects for communication, we mean physical or symbolic artifacts available to students that can be talked about, gestured to, and manipulated in their efforts to create a shared understanding. The software uses public reference objects to provide both a framework for interpretation (resources for individual conceptual change) and a place to apply that framework (resources for converging on a shared understanding as students attempt to solve problems). That is, the representations on the computer screen influence how each student interprets the activity. 
Much of the research in cognitive science focuses on this relationship between an individuals cognition and external representations (e.g. Larkin and Simon, 1987; Zhang and Norman, 1994). However, it is our position that the representations on the computer screen also provide a shared public medium for communication about their individual interpretation. And this process of using the representations to negotiate a public interpretation, in turn, provides the impetus for the students to refine their private understanding.

This theoretical framework, which views technology as providing active and supportive resources for interaction, has direct implications for instructional design. The Distributed-Mediated Learning Model (DMLM) (Gifford, 1997) provides a practical method to envision how our theoretical framework can be translated into computer mediated learning activities. The DMLM is a model that outlines the possible social and technological resources for independent and collaborative learning. The model has three major classifications of resources. First, there are representational and structural resources found in the learning activities themselves. Second, there are informational resources relevant to the activity, such as a database of previous students' questions and answers ${ }^{1}$. Third, the student has access to more able others, such as the teacher or student aids, who can help the student when appropriate.

Finally, at the center of the DMLM is the student, both as an individual, and as part of a group. Students are in control of how they utilize the array of resources. In addition, the students themselves are viewed as one of their own primary resources. We believe that students' existing conceptions play a productive role in the development of expertise. Indeed, existing student conceptions are one of the most important resources that students have for making sense of new situations (Smith, et al., 1993). An important subtext of this paper is that in designing for collaboration it is important to consider how the structure and resources of the activity interact with the students' naive intuitions about the domain.

\section{Using Existing Ideas as Building Blocks for Learning}

Given the well-documented difficulties people have in reasoning probabilistically (e.g. Kahneman and Tversky, 1996; Shaughnessy, 1992), at first glance it seems futile to expect that students can build on their naive intuitions of probability to construct a more standard conception of probability. However, accepting such a fatalistic viewpoint would mean that we would invalidate one of the most important resources that students have available to them-what they already know and believe about uncertain events. Instead, we must find ways that student intuitions can be used as building blocks for the construction of knowledge, and find ways within the learning environment to support the collaborative process of assembling these building blocks into a robust, normative understanding of probability.

In prior work (Vahey, Enyedy, and Gifford, 1997) we have shown that students' ideas about probability can be viewed in terms of four interrelated aspects of normative probability theory: randomness, outcome space, probability distribution, and the role of data. By randomness we mean the non-determinability of an event, and what predictions one can make of this non-determinable mechanism. By outcome space we mean the set of all possible outcomes. By probability distribution we mean the probabilities assigned to the different outcomes in the outcome space. Finally, by the validity of data we mean how data can be used to justify conclusions, including when and how data is considered to be useful.

For the sake of coherence and space, this paper concentrates only on the students' ideas in two closely related ideas of the four interrelated aspects in our framework - the outcome space and probability distribution. Students have great difficulty in enumerating an outcome space and in differentiating between different outcomes in the outcome space. Perhaps most importantly, students frequently fail to recognize the relevance of the outcome space at all (Fischbein et. al, 1991). For example, when shown a game in which one team scores a point on five out of eight equiprobable outcomes, and another team scores on the remaining three out of eight outcomes, many students will state that this game is fair. Students often over generalize the unpredictability of events to the point where they will say all games of chance are fair

\footnotetext{
${ }^{1}$ Many schools as of yet still do not have network capabilities. We decided, therefore, to concentrate on providing the resources to support two students' face-to-face interactions around a single computer, with a mind towards eventually adding support for location independent collaboration such as the archival data sources mentioned in the DMLM.

${ }^{2}$ We Follow Pitman (1992) and use the term outcome space instead of sample space, as this seems to be more consitent with the students' intuitions.
} 


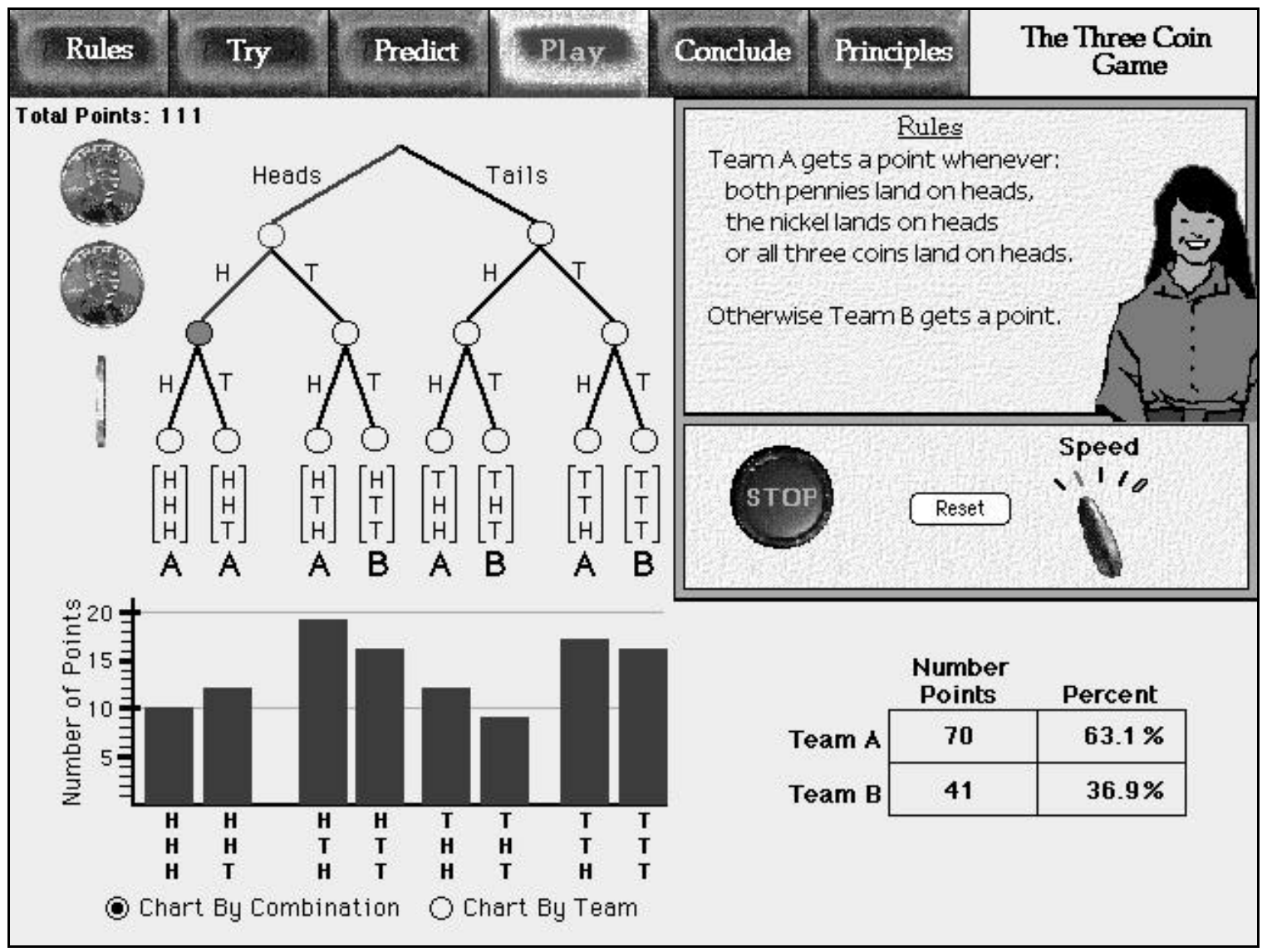

FIGURE 1 Play mode of the Three Coin Game

because what happens is all luck. However, these same students, who do not recognize the outcome space as a relevant aspect of probability, are often comfortable talking about how often classes of events are expected to occur (i.e. the probability distribution). The next section describes an activity in which students collaboratively refine their intuitions about the outcome space and connect this set of intuitions to the concept of a probability distribution.

\section{PIE: The Probability Inquiry Environment}

In PIE the students investigate the fairness of specific games of chance in the context of collaborative, guided inquiry, where each step in the investigation is designed to facilitate the type of interactions that research has shown to be most productive. Each PIE activity consists of six steps: Rules, Try, Predict, Play, Conclude and Principles.

In Rules, the software shows the students an animated introduction to the current game (the rules of the game depicted in Figure 1 will be discussed in the Qualitative Analysis Section). In Try, the students get a chance to experiment with the representations and controls of the simulation. This was done to allow the students some amount of familiarity with the environment before asking them to make predictions. In Predict, we chose questions that highlight aspects of the game that are particularly salient to a standard understanding of probability. By making collaborative predictions, students: articulate cohesive explanations in which they have some commitment; are often forced to consider alternate perspectives; and are sensitized to future events that may support the prediction or call that prediction into question (cf. Koschmann et al., 1994). In Play (see Figure 1), the software simulates the playing of the game. At this stage, PIE provides several resources and capabilities to facilitate productive collaboration. Some of the most important of these resources are: an animated probability tree that highlights the current state of the point; the partitioned outcome space that shows how each team scores; and a histogram that can toggle 
between showing the distribution across each of the outcomes or the points for each team. Each of these is a shared representational resource that can be used by the students to jointly construct an explanation of the current situation. In Conclude, the students compare their predictions to the data from the simulation. Finally, in Principles, the environment scaffolds the students to jointly articulate what they can generalize from the activity.

These activities are then followed up with real-world activities in which students flip coins, roll dice, etc. as they investigate aspects of probability without using the computer simulations. Throughout the curriculum the students also participate in full-class discussions, where each pair relates their findings from the activities, and discusses how to best determine if the game is fair. In these discussions students raise issues such as; the relative utility of the different representations, methods they used for deciding if the game is fair, and how to formalize their knowledge in the language of mathematics.

Initial work on PIE was done in laboratory studies (Enyedy, Vahey and Gifford, 1997; Vahey, Enyedy, and Gifford, 1997). These studies were used to inform the most recent version of PIE, which is the focus of this article. This recent version of PIE was implemented in two seventh grade classes in an urban middle school that serves a diverse group of students. The students were given pre- and post-tests, PIE logged all student actions on the computer, four case studies were videotaped during all the sessions, other pairs of students were taped intermittently throughout the curriculum, and the teacher was audio-taped during the sessions. On days that the students used the computers they went to a central computer lab, where there were twelve Macintosh computers. The dialogue presented in this paper is from our preliminary analysis of the video records of the classroom implementation.

\section{Quantitative Results}

In this section we will briefly present quantitative data that will address the question of, "How well did the students who used PIE develop a conceptual understanding of probability?" In the following section, qualitative data will be presented to explore how PIE supported the types of interaction and communication that fostered conceptual change.

At the end of the three week probability unit the students who used PIE $(n=45)$ and a control group ${ }^{3}$ $(n=54)$ were given a paper-and-pencil test of the probability concepts addressed by the unit. Items on this test were derived from standardized tests (National Center for Educational Statistics, 1994), suggestions from the NCTM (NCTM, 1990), items from the research literature on probabilistic reasoning (Kahneman and Tversky, 1996; Konold, 1993), and specific items created by our research group that were designed to assess the probability intuitions relevant to our instructional objectives. The score for each student was based on the percentage of correct answers on multiple choice questions (1 point each) combined with an analysis of the student's explanation of her answer. Student explanation scores (1 point each) were assigned based on whether or not the student referenced or justified their answer with an appropriate mathematical construct. We used a blind scoring process so that while scoring the test the researcher did not know to which group the student belonged.

The main finding is that the students in the PIE curriculum significantly out performed students from the control group on the post test $(\mathrm{T}=3.6, \mathrm{p}<.001)$. Students who used PIE scored an average of 14.1 on the post test, compared to the control group students who scored an average of 10.5 on the post test. This effect can be attributed to the PIE intervention because the two groups performed equally well on a pre-test administered prior to the unit $(\mathrm{T}=.21, \mathrm{p}>.5)$.

\section{Qualitative Analysis}

Given that the students in the PIE curriculum were more successful at learning basic probability, this section examines two case studies of how PIE prompted and supported social interactions that may have contributed to this success. First, we discuss a change to the structure of the environment that made the software more active in scaffolding and prompting certain classes of productive interactions. Second, we

\footnotetext{
${ }^{3}$ The control group consisted of two classes taught by the same teacher as the PIE group. The control group's unit covered the same topics as the PIE group, but was taught in the traditional manner for this school. The traditional method in this case did not use a computer but instead had the students play and analyze a number of games of chance.
} 
examine a change we made to a specific representational resource, which made this resource more accessible and useful to the students as they investigated probability.

The two case studies both occurred in one activity, the Three Coin Game. In this game two teams, Team A and Team B, score points based on the outcome of three coin flips. Team A scores a point on five of the eight possible outcomes, and Team B scores a point for three of the possible outcomes. Because each outcome is equally likely and Team A scores on more outcomes than Team B, this game is unfair in favor of Team A. The animated probability tree and dynamic histogram were both on the screen during the entire simulation (see Figure 1).

\section{Prompting Mathematical Discourse: Observation Notes}

In the laboratory study of PIE, several types of behaviors were evoked during the simulation (Play). Unfortunately, not all of these behaviors led to productive learning interactions. During the most productive (and most rare) interactions, students compared the current state of the simulation to their predictions, and attempted to formalize their understanding. At other times, however, students would become "caught up" in the simulation, excitedly watching each point and cheering the different teams on, without comparing their expectations to the results, and without attempting to formalize their understanding of the situation. Finally, students would sometimes start the simulation, become detached, and start talking "off task" (for instance, discussing recent movies). These students only turned their attention back to the environment when the simulation was completed. We suspected that the problem was that we only provided very limited ways for the student to participate with the simulation. This led us to search for ways in which we could keep students involved and scaffold productive interactions that would help students reconsider their predictions, refine their ideas, and formalize their understandings.

The extensive literature on prompting provided a starting place for such a search. Prompting has been shown to be productive in helping groups of students to become more expert readers (Brown and Palinscar, 1989), has been shown to help individual students create self-explanations while solving science problems (Chi, deLeeuw, Chiu, and LaVancher, 1994), and has been shown to help foster reflection for students engaged in computer-based activities (Davis and Linn, in preparation; Scardemelia and Bereiter, 1991). However, such studies have focused on prompting's effect on individuals cognition, but have not investigated the types of social interactions between two students that result from computer-based prompts. We chose to investigate the role of the computer as an active participant in student discourse, which seeded student conversations with questions and ideas that highlight important aspects of the learning activities. Toward this end PIE was modified so as to stop the simulation at strategic points and have the students enter observation notes. These observation notes were intended to stop the flow of the activity, draw the students' attention to salient aspects of the simulation, and provide a forum for the students to create and articulate cohesive explanations about the current situation. For example, after every two games, PIE would ask the students "Do you think the game is fair or unfair now?", and would provide a sentence starter: "We think the game is because

Preliminary analysis shows that the observation notes did prompt students to reevaluate their predictions and attempt to refine their understandings, even when students did not enter any text in response to the observation notes (a traditional measure for evaluating the effectiveness of prompts). This can be seen in Figure 2, where although $\mathbf{J}$ and $\mathrm{P}$ did not respond to the prompt by entering an observation note, simply stopping the simulation and posing a question created the opportunity for a long, productive interaction that lasted throughout the next simulation. Student $\mathrm{J}$ first responded to the question of fairness by stating that the game was, indeed, unfair, but instead of Team B winning more (as he originally predicted), Team A was winning more. They then started the game again, and $\mathrm{P}$ continued the conversation, stating that he expected that Team A would win more. In this example we see that the observation note provided an impetus for a conversation that allowed the two students to contrast the prior, typed predictions to the results of the simulation, and allowed $\mathrm{P}$ to state his disagreement with the original predictions. By stopping the flow of the activity, the computer created a space for interaction and seeded the dialogue with the possibility of critically re-evaluating their original predictions. 


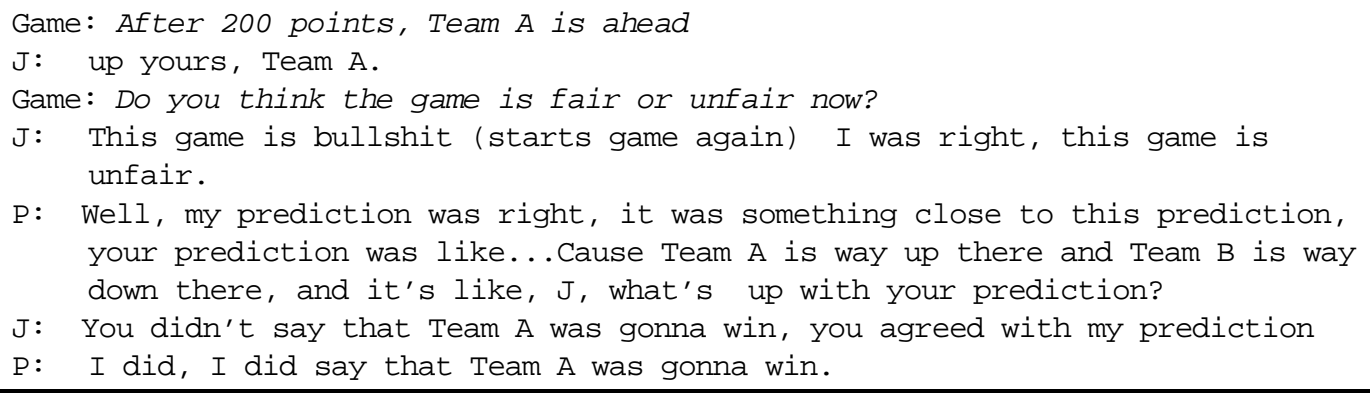

FIGURE 2: $\mathrm{J}$ and $\mathrm{P}$ comparing their predictions to the simulation.

Our preliminary analysis also shows that there were times when students took advantage of the opportunities created by the observation notes in order to refine and formalize their understanding of the situation. In Figure 3 we see the initial predictions and then the observation note of two students, Q and X, as they attempt to determine if the three-coin game is fair. In their predictions they first state that the game is fair, even while explicitly stating that Team A has more outcomes than Team B. They later state that Team A will score more points than Team B because Team A has more of a "variety". However, in their observation note we see that these ideas have been refined to include specific references to the outcome space, including the number of outcomes that score a point for each team.

These preliminary findings suggest that employing technology as an active agent that stops the flow of student activity in order to seed productive student conversation can be a productive mechanism for helping students to reflect upon and formalize their understandings. Not only can stopping the flow of activity prevent students from getting too caught up in "cheering" during the PIE simulations, but such interactions can structure discourse by presenting students with the opportunity to make their thinking visible to each other, structure their responses into appropriate forms (e.g. a claim and evidence for that claim), clear up ambiguities, and attempt to come to a negotiated understanding of the situation. And because technology was employed as an active agent that seeded discourse, it was possible to seed such interactions at the time that the most relevant evidence was readily available (for example, by asking if a game is fair immediately after a game to 200 points has ended).

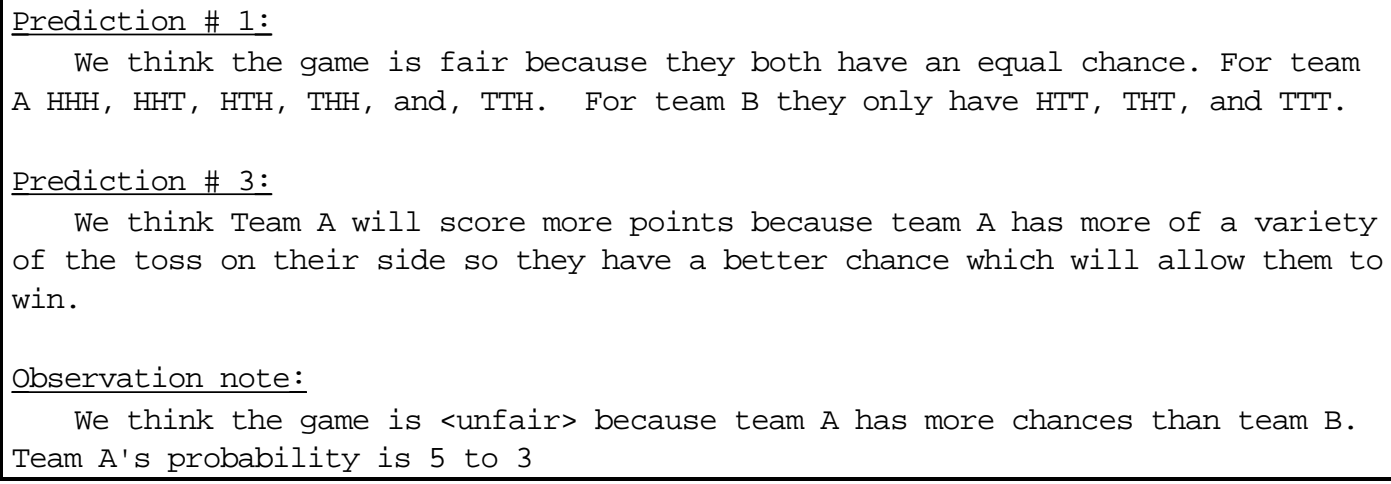

FIGURE 3: Students $\mathrm{Q}$ and $\mathrm{X}$ as they refine their thinking throughout the simulation.

\section{Supporting Mathematical Discourse: The Probability Tree}

Once begun, domain specific conversations need to be supported by a variety of resources that will allow students to articulate and revise their ideas. The effectiveness of a given computer-mediated representation to support communication and promote conceptual change will depend on its accessibility and utility to the students. However, accessibility and utility are not static - they change as the students refine and connect their intuitions. The following examples demonstrate the value of providing a few persistent representations-representations that physically do not change, but which are accessible and useful to students who are at different locations along a learning trajectory. 
As mentioned previously, students typically have many naive, non-standard intuitions about the outcome space and its relevance to reasoning probabilistically. For these students we included probability trees in each game as a visual resource that would help them organize their developing understanding of the outcome space. Probability trees can be used to spatially organize the outcome space by a series of branches and nodes that correspond to the possible outcomes of each constituent part of a compound event.

However, the students often entered the Three Coin Game with the belief that the outcome space was an irrelevant construct when deciding if the game was fair. For students at this point in their learning trajectory, the probability tree was used as a game board. That is not to say it was not useful to the students' conversations. For these students the probability tree still served as a public resource for communication, helping students when they got into linguistic trouble describing the game. At points in the activity where the students seemed to be struggling for the appropriate word or phrase to express their ideas, the students made gestures that referenced the probability tree and helped clarify the ambiguity of their verbal speech allowing the conversation to continue.

As the students' understanding progressed to the point where they accepted the relevance of the outcome space, the probability tree became more than just a crutch for a limited or ambiguous vocabulary for talking about uncertainty. The probability tree provided the students with a way to quantify the outcomes assigned to each team and compare those quantities. By the end of the activity most students had begun to use the bottom row of the probability tree as an ordered list of outcomes, counting off the combinations of outcomes that scored for each team to produce two proportions. Students then used these proportional quantities to reason about the situation and make predictions about the expected frequency of events. For example, in Figure 4 Student $\mathrm{R}$ uses the probability tree to convince her partner that the game is unfair by counting the outcomes for each team.

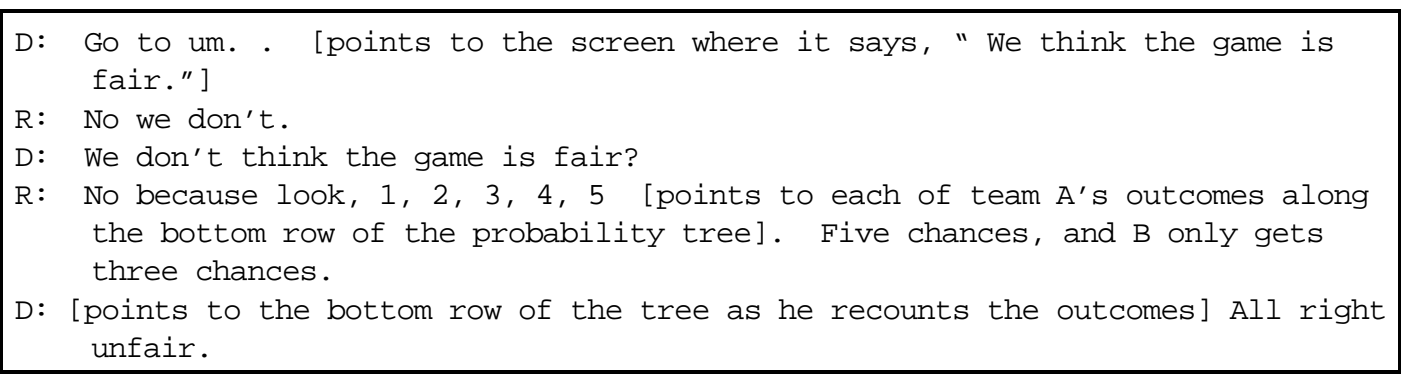

FIGURE 4: Students D and R using the tree as a list to enumerate and partition the outcome space.

In the initial laboratory study, that is where the story ended. The students progressed from using the tree as a game board to using the tree as a list. While this was an important achievement, the probability tree also supports a more sophisticated visualization and use of the outcome space that goes beyond its use as an ordered list of outcomes. The probability tree appears to encourage students to discuss their intuitions and rationale for these intuitions regarding the relationship between the outcome space and the expected probability distribution. In more complex probabilistic settings, in which all the outcomes are not equally likely, this integration of intuitions becomes essential because simply counting up outcomes will no longer correctly determine the expected probability distribution.

In the laboratory study, however, students exclusively relied on the counting strategy to decide if the game was fair and displayed no signs that they considered the frequency at which intermediate events occurred as relevant to the solution. Therefore, we took the static representation of the probability tree and added animation. (In the laboratory study, as an event happened, such as the first coin landing on heads, the probability tree would blink that branch of the tree). By animating a token along the tree, we hoped to highlight the connection between the outcome space and the probability distribution and help the students discuss the intermediate events that correspond to the constituent parts of the compound event. We predicted that this would help the students to perceive the process by which a compound event occurs and consider the frequency with which each individual constituent event occurs.

Preliminary analysis suggests that we had limited success. While the counting strategy was still the dominant way to use the tree and envision the outcome space, we did see some encouraging uses of the tree that may indicate that the students were more aware of the intermediate stages of the three coin flips and 
their relationship to the outcome space. For example in Figure 5, two adjacent pairs started to compare their ideas about what they thought was going to happen during the simulation. Student S points out that the $\mathrm{HH}$ intersection of the probability tree is important to their analysis, because if the first two flips are heads, it does not matter what the third coin lands on, team A wins either way (this state is re-created in Figure 1).

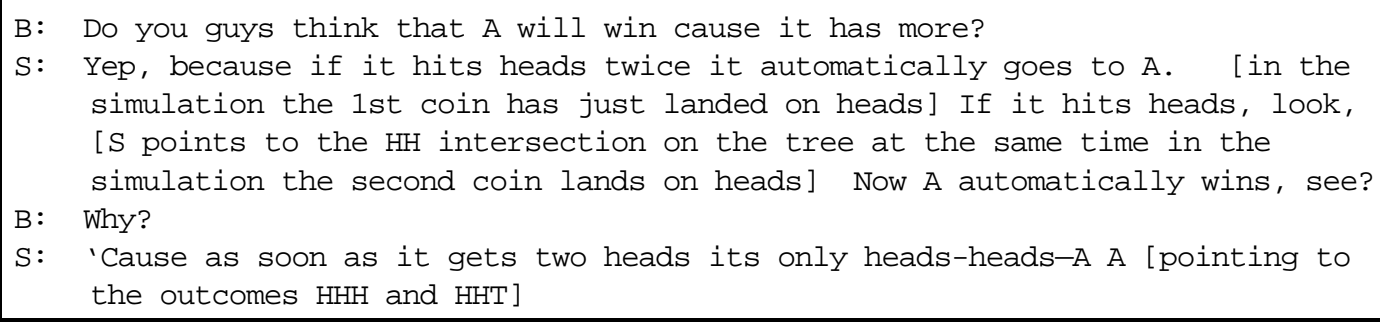

FIGURE 5: Students discussing strategic intersections of the probability tree.

The students, in this example, used the tree as a resource in their discussion of why having more outcomes matters. That is, they used the tree to help them connect their new found concept of the outcome space to their existing ideas about the frequency of events (i.e. a probability distribution).

The progression in this case-from using the tree as a game board, to using the tree as a list, finally to using the tree as way to connect previously disconnected ideas-illustrates the importance of stable and persistent representations. The tree, although unchanged physically, changed function as the students' understanding progressed from isolated intuitions about specific aspects of probability to a coherent normative understanding of the outcome space and probability distribution.

Further, this data suggests that animation may play a role in extending a representation's utility across the different stages of a students learning trajectory in our environment. The value added in this case was that the animated tree supported a different type of conversation than the static probability tree. The animated probability tree supported students in their efforts to talk about the connections between their ideas.

This can be seen as a new take on an old story. Most studies about diagrammatic reasoning focus on individuals and how animated representation (and representations in general) can be used to suggest and constrain a student's interpretation. Here we have examined how animated representations support conversations among individuals. In addition, we have looked at how multiple interpretations of a single representation can actually contribute to the learning process.

\section{Conclusion}

This paper highlights the importance of considering the process of student-to-student, face-to-face interaction when designing educational software. In our introduction we stated that educational software should create an environment where students are active in their learning by reflecting on, modifying and articulating their understanding. It is our position that this can be accomplished successfully when the software is designed to foster and support domain specific conversations.

The two case studies of students using PIE begin to explore how the rich representational resources available in computer-mediated activities can be used to support collaboration. In the first case we showed how the computer can be an active participant in the interaction by using symbolic representations and text to focus the students attention and discussion on selected features of the activity. The second case study showed how graphical representations can be used as supportive resources for communication and problem solving. The dynamic probability tree helped to make the abstract structure of the outcome space visible to the students and allowed them to use the representation as a prop in their discussions in which they articulated and refined their intuitions.

Acknowledgments

This research is funded in part by grants from the U.C. Urban Community-School Collaborative and from and the Berkeley chapter of Sigma Xi. We would like to thank the members of the InterActiveMedia Study group, and Rogers Hall for their comments. We would also like to thank Jesse Ragent for his help in the design and implementation of PIE in his classroom. 


\section{References}

Brown, A. and Palincsar, A. (1989). Guided, Cooperative Learning, and Individual Knowledge Acquisition. In Knowing, Learning, and Instruction, Resnick (Ed.) Hillsdale, NJ.

Chi, M., Bassock, M., Lewis, M. Reimann, P. and Glaser, R. (1989). Self-explanations: How students study and use examples in learning to solve problems. Cognitive Science, 13, 145-182.

Davis, E.A. and Linn, M.C. (in preparation). Scaffolding Students' Knowledge Integration through Prompts for Reflection.

diSessa, A. (1997). Personal Communication.

Enyedy, N., Vahey, P., and Gifford, B. (1997). Designing Interactions for Guided Inquiry Learning Environments. In Design of Computing Systems, (pp. 157-160). Sith, Salvensy, Koubek (Eds). Amsterdam, The Netherlands: Elsevier.

Fischbein, E., Nello, M., and Marino, M. (1991). Factors affecting probabilistic judgements in children and adolescents. Educational Studies in Mathematics 22: 523-549.

Geertz, C. (1973). The Interpretation of Culture. New York, NY: Basic Books.

Gifford, B. R. (1997). Toward a New Instructional Architecture for Distributed Learning Environments. Paper presented to the 1997 Annual Meeting of the American Educational Research Association, Chicago, April 1997.

Hutchins, E. (1995). Cognition in the Wild., (pp. 49-116). Cambridge, MA: MIT Press.

Kahneman, D., and Tversky, A. (1996). On the Reality of Cognitive Illusions. Psychological Review, v. 103, n. 3, 582-591.

Konold, C., Pollatsek, A., Well, A., Lohmeier, J., and Lipson, D. (1993). Inconsistencies in students reasoning about probability. Journal for Research in Mathematics Education. 1993, v24, pp. 392-414.

Koschmann, T.D., Myers, A.C., Feltovich, P.J., Barrows, H.S. (1994). Using Technology to Assist in Realizing Effective Learning and Instruction: A principled approach to the use of computers in collaborative learning. The Journal of the Learning Sciences, 3(3), 227-264.

Larkin, J. and Simon, H. (1987). Why a diagram is (sometimes) worth ten thousand words. Cognitive Science, 11(1), (pp. 65-100).

Lave, J. (1992). Word Problems: a microcosm of theories of learning. In Context and Cognition: Ways of Learning and Knowing, Light and Bull (Eds.). Harverster Wheatsheaf, Hertfordshire, England.

National Center for Educational Statistics (1994). NAEP 1992 Trends in Academic Progress. Washington, DC: Office of Educational Improvement and Research, US Department of Education, US Government Printing Office.

National Council of Teachers of Mathematics. (1981). Teaching Statistics and Probability, Shulte and Smart (Eds.). Reston, VA: National Council of Teachers of Mathematics

Pea, R. (1993). Practices of distributed intelligence and designs for education. In G. Salomon (Ed.) Distributed Cogntitions: Psychological and Educational considerations, (pp. 47-87). New York, NY: Cambridge University Press.

Pea, R. (1994). Seeing What We Build Together: Distributed Multimedia Learning Environments for Transformative Communications. The Journal of the Learning Sciences, 3(3), 219-225.

Pitman, J. (1993). Probability. Springer-Verlag, New York.

Scardamalia, M., and Bereiter, C. (1991). Higher Levels of Agency for Children in Knowledge Building: A Challenge for the Design of New Knowledge Media. The Journal of the Learning Sciences, 1(1), 3768.

Shaughnessy, J.M. (1992). Research in Probability and Statistics: Reflections and Directions. In Handbook of Research on Mathematics Teaching and Learning, D. Grouws (Ed), pp. 465-493.

Smith, J.P., diSessa, A., and Roschelle, J. (1993). Misconceptions Reconceived: A constructivist analysis of knowledge in transition. The Journal of the Learning Sciences, 3(2), 115-163.

Vahey, P. (1996) How Students Conceptions of Fairness Informed the Design of an Interactive Instructional Probability Environment. Presented at the American Education Researchers Association annual conference, New York, NY.

Vahey, P., Enyedy, N., and Gifford, B. (1997). Beyond Representativeness: Productive intuitions about probability. Proceedings of the Nineteenth Annual Conference of the Cognitive Science Society, M. Shafto and P. Langley (Eds.), pp. 769-774, LEA. 
Zhang, J. and Norman, D. (1994). Representations in distributed cognitive tasks. Cognitive Science, 18(1), (pp. 87-122).

\section{Authors' Addresses}

enyedy@socrates.berkeley.edu

pvaheyesocrates.berkeley.edu

bgiffordesocrates.berkeley.edu 